\title{
ARTICLE
}

Clinical Studies

\section{Pharmacokinetic-pharmacodynamic guided optimisation of dose and schedule of CGM097, an HDM2 inhibitor, in preclinical and clinical studies}

\author{
Sebastian Bauer (D) ${ }^{1}$, George D. Demetri ${ }^{2}$, Ensar Halilovic ${ }^{3}$, Reinhard Dummer ${ }^{4}$, Christophe Meille ${ }^{5}$, Daniel S. W. Tan ${ }^{6}$, \\ Nelson Guerreiro ${ }^{5,8}$, Astrid Jullion ${ }^{5}$, Stephane Ferretti ${ }^{5}$, Sebastien Jeay ${ }^{5,9}$, Laurence Van Bree ${ }^{5}$, Florence Hourcade-Potelleret ${ }^{5}$, \\ Jens U. Wuerthner ${ }^{5,10}$, Claire Fabre ${ }^{5}$ and Philippe A. Cassier (D) $^{7}$
}

\begin{abstract}
BACKGROUND: CGM097 inhibits the p53-HDM2 interaction leading to downstream p53 activation. Preclinical in vivo studies support clinical exploration while providing preliminary evidence for dosing regimens. This first-in-human phase I study aimed at assessing the safety, MTD, PK/PD and preliminary antitumor activity of CGM097 in advanced solid tumour patients (NCT01760525). METHODS: Fifty-one patients received oral treatment with CGM097 10-400 mg 3qw $(n=31)$ or 300-700 mg 3qw 2 weeks on/ 1 week off $(n=20)$. Choice of dose regimen was guided by PD biomarkers, and quantitative models describing the effect of CGM097 on circulating platelet and PD kinetics.

RESULTS: No dose-limiting toxicities were reported in any regimens. The most common treatment-related grade $3 / 4$ AEs were haematologic events. PK/PD models well described the time course of platelet and serum GDF-15 changes, providing a tool to predict response to CGM097 for dose-limiting thrombocytopenia and GDF-15 biomarker. The disease control rate was 39\%, including one partial response and 19 patients in stable disease. Twenty patients had a cumulative treatment duration of $>16$ weeks, with eight patients on treatment for $>32$ weeks. The MTD was not determined.

CONCLUSIONS: Despite delayed-onset thrombocytopenia frequently observed, the tolerability of CGM097 appears manageable. This study provided insights on dosing optimisation for next-generation HDM2 inhibitors.

TRANSLATIONAL RELEVANCE: Haematologic toxicity with delayed thrombocytopenia is a well-known on-target effect of HDM2 inhibitors. Here we have developed a PK/PD guided approach to optimise the dose and schedule of CGM097, a novel HDM2 inhibitor, using exposure, platelets and GDF-15, a known p53 downstream target to predict patients at higher risk to develop thrombocytopenia. While CGM097 had shown limited activity, with disease control rate of 39\% and only one patient in partial response, the preliminary data from the first-in-human escalation study together with the PK/PD modeling provide important insights on how to optimize dosing of next generation HDM2 inhibitors to mitigate hematologic toxicity.
\end{abstract}

British Journal of Cancer (2021) 125:687-698; https://doi.org/10.1038/s41416-021-01444-4

\section{INTRODUCTION}

Tumour suppressor p53 is a transcription factor that controls the expression of a myriad of target genes involved in DNA repair, apoptosis, and cell-cycle arrest, which are all important processes counteracting the malignant growth of tumours. TP53 is one of the most commonly mutated genes in human cancer. Approximately $50 \%$ of all human cancers harbour TP53 mutations. In cancers where the TP53 gene is not mutated, the function of the p53 pathway is often suppressed through mechanisms that affect the stability and activity of the p53 protein. One such mechanism is overexpression or deregulation of MDM2. MDM2, for which the human orthologue is also known as $\mathrm{HDM} 2$, is an E3 ubiquitin ligase, which by direct binding negatively regulates p53 through ubiquitination and subsequent proteasomal degradation. ${ }^{1}$

CGM097 inhibits the p53-HDM2 interaction which protects p53 from degradation, subsequently leading to its accumulation and activation resulting in stimulation of downstream effector pathways that induce cell-cycle arrest and/or, apoptosis through transcriptional activation of cell-cycle inhibitory genes (e.g. CDKN1A), and pro-apoptotic genes (e.g. PUMA and NOXA). ${ }^{2-4}$

\footnotetext{
${ }^{1}$ Department of Medical Oncology, Sarcoma Center, West German Cancer Center, University of Duisburg-Essen, Duisburg-Essen, Germany; ${ }^{2}$ Dana-Farber Cancer Institute and Ludwig Center at Harvard Medical School, Boston, MA, USA; ${ }^{3}$ Novartis Institutes for BioMedical Research (NIBR), Cambridge, MA, USA; ${ }^{4}$ University Hospital Zurich, Zurich, Switzerland; ${ }^{5}$ Novartis Institutes for BioMedical Research (NIBR), Basel, Switzerland; ${ }^{6}$ National Cancer Center Singapore, Singapore, Singapore and ${ }^{7}$ Department of Medical Oncology, Centre Léon Bérard, Lyon, France
}

Correspondence: Sebastian Bauer (sebastian.bauer@uk-essen.de)

${ }^{8}$ Present address: F. Hoffmann-La Roche AG, Basel, Switzerland

${ }^{9}$ Present address: Idorsia Pharmaceuticals Ltd, Allschwil, Switzerland

${ }^{10}$ Present address: ADC Therapeutics, Epalinges, Switzerland

Received: 23 November 2020 Revised: 26 March 2021 Accepted: 17 May 2021

Published online: 17 June 2021 
HDM201 inhibits the growth, in vitro and in vivo, of tumour models with functional wild-type p53 derived from a variety of cancer types. ${ }^{2-5}$ Here, we present the preclinical validation and the results of the first-in-human phase I study conducted to determine the safety, maximum tolerated dose (MTD), pharmacokinetic/ pharmacodynamic (PK/PD), and preliminary antitumor activity of CGM097 in patients with advanced solid tumours. In addition, we describe the development of an integrated PK/PD modelling approach to characterise the temporal dose-response relationships of drug action on platelets and serum growth differentiation factor-15 (GDF-15) as a downstream marker of p53 pathway activation; predict platelet response and incidence rates of thrombocytopenia; and identify associations between GDF-15 induction and risk of developing delayed thrombocytopenia. Several tumours with high a priori p53 wild-type status, including melanoma, soft-tissue sarcoma, osteosarcoma, renal cell carcinoma, and proximal colorectal tumours, were investigated. ${ }^{6-10}$

\section{METHODS}

Preclinical studies

Animal study design. All animal experiments were performed according to procedures covered by permit number BS-1975 issued by the Cantonal Veterinary Office, Basel, Switzerland, and in strict adherence to the Federal Animal Protection Act and the Federal Animal Protection Code. Female athymic nude mice (Hsd: Athymic Nude-Foxn1 nu, 7-8 weeks old, 20-22 g) were obtained from Envigo (Germany). All animals were housed in a pathogencontrolled environment with access to food and water ad libitum, and were identified with transponders.

CGM097 was freshly dissolved in $0.5 \%$ hydroxypropyl methylcellulose for PO administration at $10 \mathrm{~mL} / \mathrm{kg}$. For PK/PD experiments, animals were randomised into groups of three, and tissue samples were collected at $0,1,3,8,16,24$ and $48 \mathrm{~h}$. At the times indicated, animals were anaesthetised by exposure to $2-3 \% \mathrm{v} / \mathrm{v}$ isoflurane in medical oxygen. For efficacy experiments, animals were randomised into groups $(n=6)$ for a mean tumour size of $100 \mathrm{~mm}^{3}$, and CGM097 was administered at several doses according to the respective dosing regimen for 14 days.

Assessments. Subcutaneous SJSA-1, 778 and LP6 tumours were induced by injecting tumour cells expanded in vitro into the right flank of nude mice. For (PK)/(PD) experiments, CGM097 was injected once at 50 or $100 \mathrm{mg} / \mathrm{kg}$. After collecting blood samples for determining plasma concentrations of CGM097, the animals were sacrificed before they recovered from anaesthesia. The tumours were excised, weighed and rapidly frozen in liquid nitrogen. Later, they were cryogenically dry pulverised using the CryoPrep $^{\mathrm{TM}}$ system (model CP-02, Covaris).

Tumour response was reported with the measures of tumour volumes from the treatment start. Concentrations of CGM097 in plasma were determined simultaneously by ultra-performance liquid chromatography-tandem mass spectrometry. The total RNA was purified from cell pellets using the QIAshredder (cat \# 79654, Qiagen) and RNeasy Mini Kit (74106, Qiagen) according to the manufacturer's instructions, with the exception that no DNA digestion was performed. Total RNA was quantitated using the spectrophotometer ND-1000 Nanodrop ${ }^{\circ}$. Quantitative reverse transcriptase PCR (qRT-PCR) was set up in triplicate per sample using either the One-Step RT qPCR Master Mix Plus (RT-QPRT-032X, Eurogentec) or the $\mathrm{iTaq}^{\mathrm{TM}}$ Universal Probes One-Step Kit (\#1725141), with primers from TaqMan Gene Expression assays $\left(20 \times\right.$ probe dye $\mathrm{FAM}^{\mathrm{TM}}$ [or VIC]-TAMRA [or MGB]; Applied Biosystems), including control primers (GUS- $\beta$ 4310888E-1012026) and primers for the target genes (CDKN1A Hs00355782_m1; BBC3 Hs00248075_m1; MDM2 Hs01066930_m1). Alternatively, we performed a multiplex gene expression analysis on 104 p53 target genes with the NanoString assay.
Clinical study

Study design. This phase I, multicenter, open-label study (NCT01760525) evaluated CGM097 as an oral single-agent administered in a continuous three times a week (3qw) schedule in patients with advanced solid tumours (regimen 1). The protocol allowed switching to one or more of three alternative regimens; of these, the intermittent dosing regimen of 3qw for 2 weeks on/ 1 week off was investigated. The study was conducted in accordance with the Declaration of Helsinki and Good Clinical Practice and approved by Institutional review boards. All patients provided written informed consent.

Patients. Eligible patients were $\geq 18$ years old, had locally advanced or metastatic solid tumours (including but not restricted to melanoma, proximal colorectal cancer, soft-tissue sarcoma, osteosarcoma or renal cell carcinoma) with evidence of disease progression, and had failed or been ineligible for standard-of-care therapy with a World Health Organisation performance status of 0 to 2 and adequate organ function. All patients were required to have TP53 ${ }^{w t}$ status or, at a minimum, no mutations in exons 5, 6, 7 and 8 determined locally whenever applicable and/or assessed centrally. TP53 status could be determined on archival samples no older than 36 months at study entry. Patients having a prior treatment with compounds with the same mechanism of action as CGM097 were excluded. Patients with central nervous system metastatic lesions or concurrent other malignancy or clinically significant cardiac disease or abnormal laboratory findings were excluded.

Assessments. The primary objective was to determine the MTD and/or identify the recommended dose for expansion of CGM097 for the daily and intermittent dosing regimens.

Safety was assessed according to the National Cancer Institute Common Terminology Criteria for Adverse Events (NCI-CTCAE) v4.03. Antitumor activity was assessed by Response Evaluation Criteria In Solid Tumours (RECIST) v1.1. Serial blood samples were collected throughout cycle 1, then sparse samples on day 1 of cycle 2 and predose samples on subsequent cycles, to determine plasma CGM097 concentrations. Fresh biopsies were collected on day 8 of cycle 1 along with a matching PK sample $(6 \mathrm{~h} \pm 2 \mathrm{~h}$ postdose). Plasma concentrations of CGM097 were measured using a validated liquid chromatography-tandem mass spectrophotometry approach, with a lower limit of quantification of $\sim 1 \mathrm{ng} / \mathrm{mL}$.

Pharmacodynamic tumour marker evaluation

Serum growth differentiation factor-15 (GDF-15) was measured using the Quantikine ELISA kit (\#DGD150, R\&D Systems). Briefly, serum samples were diluted 1:4 and transferred to pre-coated plates. The assay was run with an 8-point standard curve, and absorbance was read at $450 \mathrm{nM}$. All samples were assayed in duplicate, and mean values were reported if within range $(25-45,000 \mathrm{pg} / \mathrm{mL})$. A coefficient of variation $(C V \%)<20$ between duplicates was considered acceptable. The fold increase in GDF-15 at $24 \mathrm{~h}$ post-dose CGM097 (cycle 1, day 2) was measured relative to baseline.

\section{Statistical analysis}

A Bayesian Logistic Regression Method (BLRM) employing the Escalation With Overdose Control (EWOC) was used during the escalation phase for the selection of doses. Determination of the MTD during escalation was based upon the estimation of the probability of DLT in cycle 1 in patients belonging to the dosedetermining set. Dose escalation decisions were based on a clinical synthesis of all relevant available data (toxicity, PK, and PD information) together with the DLT information.

Antitumor efficacy was assessed using computerised tomography $(\mathrm{CT}) /$ magnetic resonance imaging assessment at baseline and every 8 weeks until disease progression or the initiation of 
subsequent antineoplastic therapy or death, whichever occurred first. In addition, the criteria for determining confirmed partial response (PR) or complete response (CR) were required to be present for at least 4 weeks. Demographic and other baseline data and PK/PD parameters were summarised using descriptive statistics. ${ }^{11}$ PK parameters were estimated using noncompartmental methods (Phoenix ${ }^{\circledast}$ Pharsight, Mountain View, California, USA).

Pharmacokinetic and pharmacodynamic modelling

A total of 1919 observations (924 plasma PK, 717 platelet, and 278 GDF-15 measurements) and 20 platelet transfusion events derived from 46 patients were included in the dataset (data cutoff, January 2016). A nonlinear mixed-effect modelling approach ${ }^{12}$ was used to formulate the models describing the PK, serum GDF-15 and platelet time-course relationship with CGM097 treatment. We used the stochastic approximation of the expectationmaximisation (SAEM) algorithm ${ }^{13}$ implemented in the Monolix software (version 4.3.2, Lixoft) to estimate the fixed (populationlevel typical values) and random (inter-individual variability) effects for each model parameter and a residual error.

In brief, the PK properties of CGM097 was described by a 2compartment model with a delayed first-order absorption and a linear elimination. A semi-mechanistic model mimicking hematopoietic cell maturation and regulation was developed to describe the time course of platelet kinetics and drug action on immature cells and modified from Friberg et al. ${ }^{14}$ The change in serum GDF15 in response to CGM097 was described by a type III indirect response PD model with drug stimulation of GDF-15 production as previously described. ${ }^{15,16}$ A schematic diagram of the PK and PK/ PD models for platelets (thrombocytopenia) and for GDF-15 timecourse relationship is presented in Supplementary Fig. S1. The equations for the respective models are provided in the Supplementary Material (Supplementary Data).

Models were evaluated based on the change in the Akaike's information criterion (AIC) value, the convergence of the SAEM algorithm, the precision of the parameter estimates (NLME derived $\%$ relative standard error [RSE\%]) and decreases in IIV and residual variability. Diagnostic plots were used to evaluate model adequacy, including evaluation of observations versus population and individual predicted (IPRED), and the distributions of conditional weighted residuals (CWRES) or normalised prediction distribution error (NPDE) versus time/observations or over time plots (Supplementary Figs. S2-S4). The predictive performance of the final models was evaluated by simulating 500 datasets using parameter estimates (fixed and random effects) and plotting a visual predictive check (Supplementary Fig. S5). For serum GDF-15, serum concentration levels above the upper limit of quantification $(>50,000 \mathrm{pg} / \mathrm{mL}$ ) were handled as right-censored data.

\section{RESULTS}

Identification of the three times a week (3qw) dosing regimen in preclinical, PK, PD, efficacy study

NanoString analysis with selected known p53-regulated genes was performed in SJSA-1 tumours from animals treated with a single $50 \mathrm{mg} / \mathrm{kg}$ dose of CGM097 to identify the most highly upand downregulated p53 target genes. Results revealed the activation of p53 transcriptional function, with a 12-fold increase in BCL2-binding component 3 (BBC3), a pro-apoptotic member of the $\mathrm{Bcl}-2$ protein family, also known as p53-upregulated modulator of apoptosis (PUMA). Cyclin-dependent kinase inhibitor 1 (CDKN1A), also known as p21, was upregulated by a factor of 10 . GDF-15, also known as MIC1, and HDM2 were upregulated, albeit to a lesser extent. On the other hand, the activation of p53 caused a significant decrease in mRNA expression of E2F1 and TP73, while there was no effect on TP63 expression. Genes involved in cell-cycle regulation (CDK1, CDK2, CCNE1, GTSE1) and DNA repair (ATM, BRCA1, CHEK1), and BCL-2 were also downregulated (Fig. 1a).

To characterise the PK/PD relationship, we applied the theory of "free-drug hypothesis", which states that the pharmacological activity of a drug is determined by the unbound drug concentration. The estimated unbound fractions in plasma were $0.193 \% \pm$ $0.0567 \%$ for the mouse. Treatment with CGM097 at $100 \mathrm{mg} / \mathrm{kg}$ in SJSA-1 tumour-bearing mice showed that the unbound drug concentration in plasma (plasma protein binding $=99.8 \%$ in mouse) stayed above the in vitro biochemical inhibitory concentration $90 \%$ (IC90) (14 nM) for the first $24 \mathrm{~h}^{17}$ and such sustained exposure highly induced p21 (maximum effect Emax $=41$-fold) and PUMA, (Emax =19-fold) (Fig. 1b). HDM2 had the lowest increase in expression among these three genes. As previously published, ${ }^{18}$ the time to reach the Emax is delayed compared to plasma Cmax as p53 target genes accumulate as long as unbound drug concentration in plasma stay above the threshold and p53 remains activated. Once the unbound drug concentration decreased below the $I_{90}$, the pharmacological activity dramatically decreased and reached basal levels $48 \mathrm{~h}$ post treatment (Fig. 1b).

Daily treatment of tumour-bearing mice with CGM097 dosedependently affected SJSA-1 tumour growth in vivo (Fig. 1c). When dosed daily at $100 \mathrm{mg} / \mathrm{kg}, 65 \%$ of tumour regression was reached after 10 days of treatment. When dosed daily at $50 \mathrm{mg} / \mathrm{kg}$, stable disease $(\mathrm{SD})$ was observed $(\mathrm{T} / \mathrm{C}=3 \%)$; tumour growth was reduced by $50 \%$ at the $25 \mathrm{mg} / \mathrm{kg}$ dose compared to the vehicle treatment group. Similar tumour growth inhibition and dose-response was reached in additional MDM2-amplified welldifferentiated (WDLPS) and dedifferentiated liposarcoma (DDLPS) models (Supplementary Fig. S6). Three additional dosing regimens (3qw, 2qw or 3 days on/4 days off) were also investigated, and similar regression levels were observed in comparison with the daily dosing regimen (Fig. 1d), confirming that the efficacy of CGM097 is not dosing regimen dependent. Based on a one-compartment PK model (WinNonlin/Phoenix 6.3) of mouse, PK parameters (average plasma concentrations at steady-state $\left[C_{\text {ave-ss }}\right]$ and maximum plasma concentrations at steady-state $\left.\left[C_{\text {max-ss }}\right]\right)$ were simulated for the three alternative dosing regimens in addition to the daily regimen (Fig. 1d). Among all the dosing regimens inducing similar tumour regression, the 3qw regimen was expected to result in the best therapeutic index in the clinic, as it had a lower weekly dose $(450 \mathrm{mg} / \mathrm{kg})$ and $C_{\text {ave-ss }}(27.9 \mathrm{nM})$ compared with the standard daily treatment regimen $(700 \mathrm{mg} / \mathrm{kg}$ and $37.3 \mathrm{nM})$, and a lower $C_{\text {max-ss }}(75.8 \mathrm{nM})$ compared with the 2 qw $(96.4 \mathrm{nM})$ and 3 days on/4 days off $(92.3 \mathrm{nM})$ regimens.

Treatment regimens and study population

In the clinical study, 51 patients with advanced solid tumours were enrolled in five centres across five countries. In regimen 1, 31 patients were administered CGM097 3qw continuously until disease progression at doses of $10 \mathrm{mg}(n=3), 20 \mathrm{mg}(n=4)$, $40 \mathrm{mg}(n=4), 80 \mathrm{mg}(n=4), 150 \mathrm{mg}(n=4), 300 \mathrm{mg}(n=7)$ and $400 \mathrm{mg}(n=5)$. Twenty patients were treated with the alternative regimen, 2 weeks on 11 week off at doses of $300 \mathrm{mg}(n=8), 500$ $\mathrm{mg}(n=6)$ and $700 \mathrm{mg}(n=6)$. Twenty patients $(39.2 \%)$ had a cumulative treatment duration of $>16$ weeks, with eight patients (15.7\%) reporting more than 32 weeks of treatment.

At the cutoff date (July 7, 2016), the median treatment duration for all patients was 9.7 weeks (1.7-118 weeks). All except four patients, received more than 4 weeks of treatment, with four patients (7.8\%) reporting $24-32$ weeks and eight patients (15.7\%) reporting more than 32 weeks of treatment, respectively. The mean treatment duration was the longest in the CGM097 (400 mg) regimen 1 treatment group (36.1 weeks). Demographics and patient disposition are summarised (Table 1). 
a Gene expression analyses by NanoString of p53 target genes in vivo

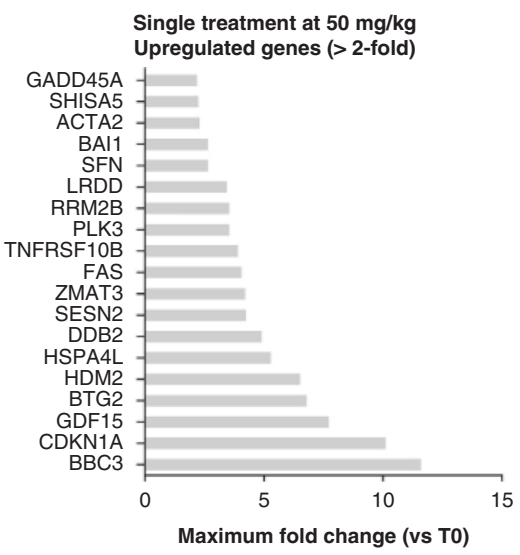

b

In vivo PK/PD relationship

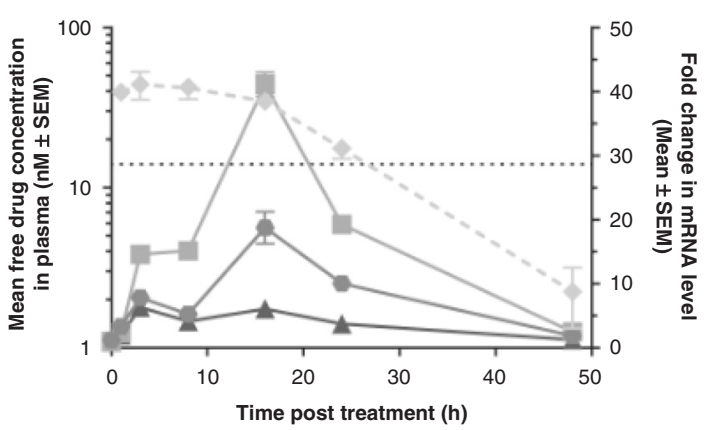

- Free drug concentration in plasma
p21 mRNA induction in tumour
-... Biochemical IC90 (TR-FRET-MDM2)
- PDM2 mRNA induction in tumour
- PUMA mRNA induction in tumour

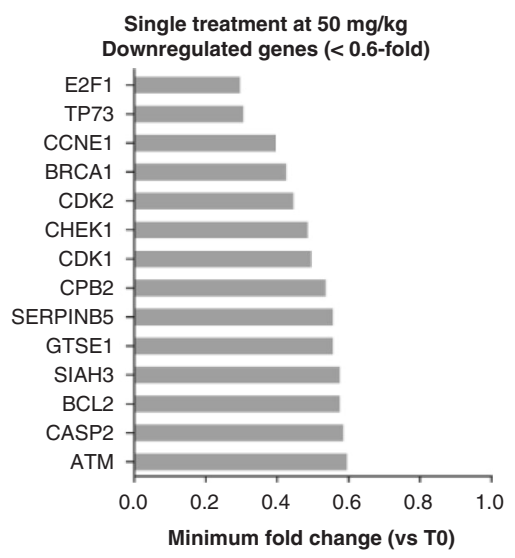

C

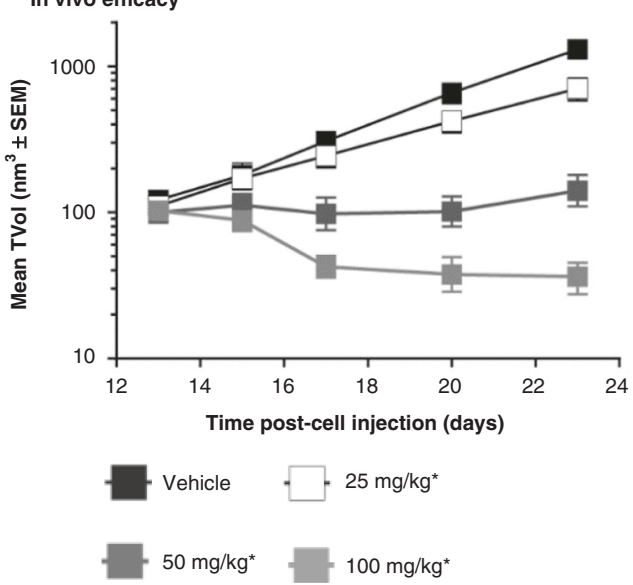

d In vivo PK modelling

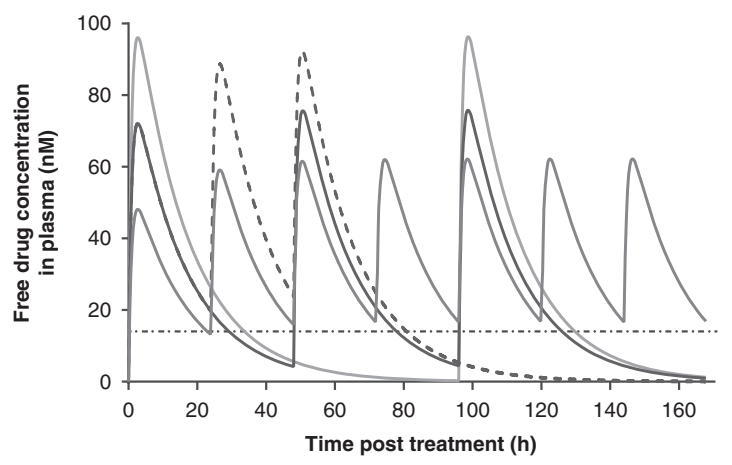

$\begin{array}{ll}\text { - Predicted } 100 \mathrm{mg} / \mathrm{kg} \mathrm{q} 24 \mathrm{~h} & \text { - Predicted } 150 \mathrm{mg} / \mathrm{kg} 3 \mathrm{qw} \\ \text { — Predicted } 200 \mathrm{mg} / \mathrm{kg} 2 \mathrm{qw} & \text { - Predicted } 150 \mathrm{mg} / \mathrm{kg} 3 \mathrm{~d} \text { on } / 4 \mathrm{~d} \text { off } \\ \text { _... Biochemical IC90 (TR-FRET-MDM2) } & \end{array}$

Fig. 1 Preclinical data in SJSA-1 tumour-bearing mice treated with CGM097. a NanoString analysis with selected known p53-regulated genes performed in SJSA-1 tumours from animals treated with a single $50 \mathrm{mg} / \mathrm{kg}$ dose of CGM097. b For PK/PD experiment, mice were randomised into groups of $n=3$ and sacrificed/sampled over a $48 \mathrm{~h}$ period of time. Unbound plasma exposure was calculated on the basis of a free fraction of $0.193 \%$ in the mouse. The black dotted lines represent the biochemical IC90. c For efficacy experiment, mice were randomised into groups of $n=6$ and CGM097 was administered at several doses according to respective dosing regimen for 14 days. Differences between the means of TVol were assessed on the endpoint using a one-way ANOVA with Dunnett's tests post hoc $(* P<0.05$ considered as significant). d One-compartment PK model (WinNonlin/Phoenix 6.3) of the mouse was used to estimate PK parameters (average plasma concentrations at steady-state $\left[C_{\text {ave-ss }}\right]$ and maximum plasma concentrations at steady-state $\left.\left[C_{\text {max-ss }}\right]\right)$ for the four dosing regimens and correlate with the observed regression. a Gene expression analyses by NanoString of p53 target genes in vivo. b In vivo PK/PD relationship. c In vivo efficacy. $\mathbf{d}$ In vivo PK modelling. 


\begin{tabular}{|c|c|c|c|c|c|c|c|c|c|c|c|}
\hline $\begin{array}{l}\text { Demographic } \\
\text { variables }\end{array}$ & $\begin{array}{l}10 \mathrm{mg} \\
\text { Reg 1, } \\
N=3\end{array}$ & $\begin{array}{l}20 \mathrm{mg} \\
\operatorname{Reg} 1, \\
N=4\end{array}$ & $\begin{array}{l}40 \mathrm{mg} \\
\text { Reg 1, } \\
N=4\end{array}$ & $\begin{array}{l}80 \mathrm{mg} \\
\text { Reg 1, } \\
N=4\end{array}$ & $\begin{array}{l}150 \mathrm{mg} \\
\operatorname{Reg} 1, \\
N=4\end{array}$ & $\begin{array}{l}300 \mathrm{mg} \\
\text { Reg 1, } \\
N=7\end{array}$ & $\begin{array}{l}400 \mathrm{mg} \\
\text { Reg 1, } \\
N=5\end{array}$ & $\begin{array}{l}300 \mathrm{mg} \\
\text { Reg } 3, \\
N=8\end{array}$ & $\begin{array}{l}500 \mathrm{mg} \\
\operatorname{Reg} 3, \\
N=6\end{array}$ & $\begin{array}{l}700 \mathrm{mg} \\
\text { Reg } 3, \\
N=6\end{array}$ & $\begin{array}{l}\text { All } \\
\text { patients, } \\
N=51\end{array}$ \\
\hline \multicolumn{12}{|l|}{ Age (years) } \\
\hline Mean (SD) & $47(8.89)$ & $50(9.13)$ & $\begin{array}{l}50.8 \\
(13.65)\end{array}$ & $59.3(5.38)$ & $44.5(7.0)$ & $58.7(7.67)$ & $56.8(12.05)$ & $50.3(12.51)$ & $56.3(11.55)$ & $56.7(8.26)$ & $53.6(10.33)$ \\
\hline \multicolumn{12}{|c|}{ Age group (years), $n$ (\%) } \\
\hline$<65$ & $3(100)$ & $4(100)$ & $3(75.0)$ & $3(75.0)$ & $4(100)$ & $5(71.4)$ & $3(60.0)$ & $7(87.5)$ & $5(83.3)$ & $5(83.3)$ & $42(82.4)$ \\
\hline$\geq 65$ & 0 & 0 & $1(25.0)$ & $1(25.0)$ & 0 & $2(28.6)$ & $2(40.0)$ & $1(12.5)$ & $1(16.7)$ & $1(16.7)$ & $9(17.6)$ \\
\hline \multicolumn{12}{|l|}{ Sex, $n(\%)$} \\
\hline Male & $1(33.3)$ & $3(75.0)$ & $3(75.0)$ & $2(50.0)$ & $1(25.0)$ & $2(28.6)$ & $2(40.0)$ & $3(37.5)$ & $2(33.3)$ & $3(50.0)$ & $22(43.1)$ \\
\hline Female & $2(66.7)$ & $1(25.0)$ & $1(25.0)$ & $2(50.0)$ & $3(75.0)$ & $5(71.4)$ & $3(60.0)$ & $5(62.5)$ & $4(66.7)$ & $3(50.0)$ & $29(56.9)$ \\
\hline \multicolumn{12}{|l|}{ WHO PS, $n(\%)$} \\
\hline 0 & $2(66.7)$ & $3(75.0)$ & $3(75.0)$ & $2(50.0)$ & $2(50.0)$ & $4(57.1)$ & $4(80.0)$ & $4(50.0)$ & $3(50.0)$ & $4(66.7)$ & $31(60.8)$ \\
\hline 1 & 0 & $1(25.0)$ & 0 & $1(25.0)$ & $2(50.0)$ & $2(28.6)$ & $1(20.0)$ & $4(50.0)$ & $3(50.0)$ & $2(33.3)$ & $16(31.4)$ \\
\hline 2 & $1(33.3)$ & 0 & $1(25.0)$ & $1(25.0)$ & 0 & $1(14.3)$ & 0 & 0 & 0 & 0 & $4(7.8)$ \\
\hline \multicolumn{12}{|c|}{ Patient disposition, $n$ (\%) } \\
\hline $\begin{array}{l}\text { Treatment } \\
\text { discontinued }\end{array}$ & $3(100)$ & $4(100)$ & $4(100)$ & $4(100)$ & $4(100)$ & $6(85.7)$ & $4(80.0)$ & $8(100)$ & $6(100)$ & $6(100)$ & $49(96.1)$ \\
\hline Treatment ongoing & 0 & 0 & 0 & 0 & 0 & $1(14.3)$ & $1(20.0)$ & 0 & 0 & 0 & $2(3.9)$ \\
\hline \multicolumn{12}{|c|}{ Primary reason for treatment end, $n(\%)$} \\
\hline Adverse event & 0 & 0 & 0 & 0 & 0 & $1(14.3)$ & $2(40.0)$ & $1(12.5)$ & 0 & $1(16.7)$ & $5(9.8)$ \\
\hline Consent withdrawn & 0 & 0 & 0 & 0 & 0 & 0 & 0 & 0 & 0 & $1(16.7)$ & $1(2.0)$ \\
\hline Death & $1(33.3)$ & 0 & $1(25.0)$ & 0 & 0 & 0 & 0 & 0 & 0 & 0 & $2(3.9)$ \\
\hline Disease progression & $2(66.7)$ & $4(100)$ & $3(75.0)$ & $4(100)$ & $4(100)$ & $5(71.4)$ & $2(40.0)$ & $7(87.5)$ & $6(100)$ & $4(66.7)$ & $41(80.4)$ \\
\hline
\end{tabular}

Reg regimen, $P S$ performance status.

Reg 1: 3qw dosing, 4-week treatment cycle, continuous; Reg 3: 3qw dosing, 3-week treatment cycle; 2 weeks on treatment and 1 week off treatment.

Clinical safety

No formal DLTs were observed during the study. However, DLTlike events reported after day 28 (cycle 1) suggested that continuous 3qw dosing was not well-tolerated, leading to delayed grade 3 or 4 thrombocytopenia. The onset of on-target myelosuppression appeared to be delayed, however, the effect was cumulative over time. Twenty-five patients experienced grade $3 \mathrm{AEs}$ and 12 patients experienced grade $4 \mathrm{AEs}$ regardless of study drug relationship. Most frequently reported AEs (any grade) were nausea $(n=24)$, thrombocytopenia $(n=22)$, anaemia, fatigue and vomiting ( $n=18$ patients each). Grade 3 AEs suspected to be study drug-related were observed in 11 patients, and 10 patients reported at least one grade $4 \mathrm{AE}$. The incidence of grade $3 / 4 \mathrm{AEs}$ suspected to be drug-related was more common at doses $\geq 300$ $\mathrm{mg}$. In addition, the incidence of delayed haematologic AEs generally increased with increasing dose on the continuous dosing regimen (3qw); however, the incidence was reduced on the alternative dosing regimen ( 2 weeks on/ 1 week off), despite higher doses of treatment.

The most common treatment-related grade 3 or 4 AEs were thrombocytopenia $(n=12)$, lymphopenia $(n=6)$, and neutropenia $(n=6)$ (Table 2$)$. The most frequently reported treatment-related AEs included thrombocytopenia $(n=22)$, nausea $(n=21)$, leukopenia $(n=14)$, vomiting $(n=13)$ and fatigue $(n=13)$ (Table 2). Overall, five patients had at least one AE leading to study drug discontinuation. Seven deaths occurred on treatment, two while receiving the study drug and five within 30 days of treatment discontinuation; none were suspected to be treatment-related.

Because of the expected haematological on-target toxicity of MDM2 inhibitors, haematologic AEs were identified as adverse events of special interest (AESI) and included neutropenia, thrombocytopenia, leucopenia, anaemia and lymphopenia. ${ }^{19}$ AESIs occurred in $64.7 \%$ of patients regardless of study drug relationship, and in $51 \%$ as suspected to be treatment-related. Thrombocytopenia was the most common AESI (22 patients, 43.1\%); all being suspected to be treatment-related; occurred more frequently at higher doses and was present in all patients on the $400 \mathrm{mg}$ dose in regimen 1. Anaemia was the next most common AESI regardless of relationship; however, the least common drug-related $\mathrm{AESI}$, with no obvious relationship to dose. Neutropenia occurred more frequently at higher doses, particularly with the $300 \mathrm{mg}$ and $400 \mathrm{mg}$ doses in the continuous 3qw dosing regimen; nearly all were considered to be treatmentrelated.

\section{Clinical efficacy}

Fifty-one patients were evaluated for efficacy every 8 weeks and/ or at the end of treatment with CGM097. Nineteen patients, ten on the continuous and nine on the alternative dosing regimen, had $\mathrm{SD}$ as the best response. One patient with malignant melanoma on CGM097 300 mg (3qw dosing continuous) had PR (Fig. 2a) and the response was ongoing after 118 weeks at the time of cutoff date. Clinical benefit was observed in 20 patients; the disease control rate was $39 \%$ (20/51 patients). No CR occurred at any dose (Table 3). The correlation between efficacy and the average CGM097 dose intensity by cycle is represented in Supplementary Fig. S7. This figure illustrates that the majority of the clinical SD (and PR) were observed in the dose intensity ranges predicted to be active in preclinical efficacy studies.

The best-percentage change from baseline by treatment group and mutation status is presented in Fig. $2 \mathrm{~b}$. Of note, nine patients included in the trial were found to have TP53 mutations after central p53 status reassessment using FoundationOne test (Fig. 2b). These patients were included in the study based on negative p53 mutation status assessed by local p53 testing (7 patients) or based on Amplichip p53 testing performed with a CRO (two patients). Six of these nine patients progressed rapidly after the study start.

Pharmacokinetics and pharmacodynamics

Oral treatment with CGM097 in the fasted state resulted in a median time to reach maximum plasma concentrations ( $\left.T_{\max }\right)$ 


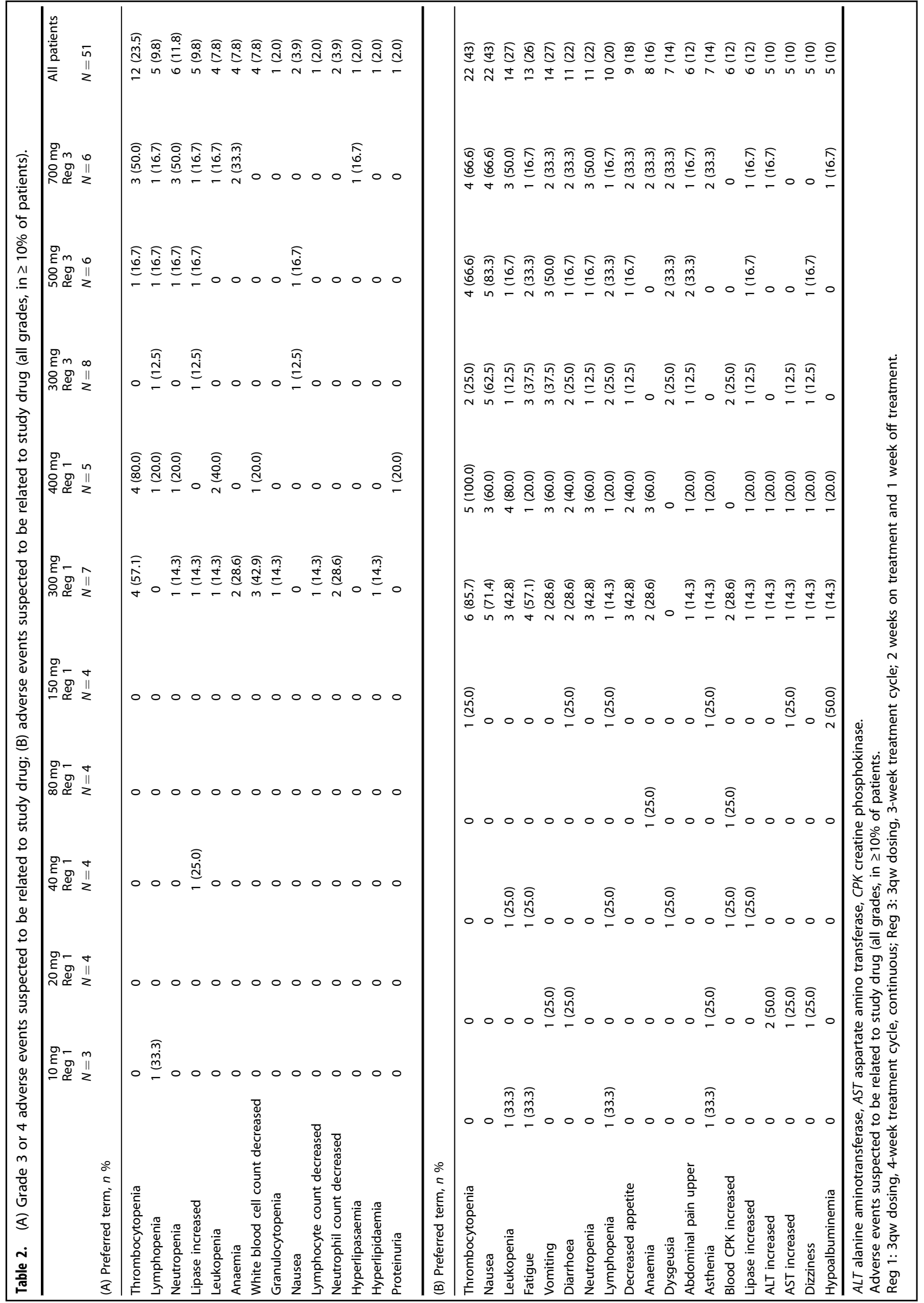



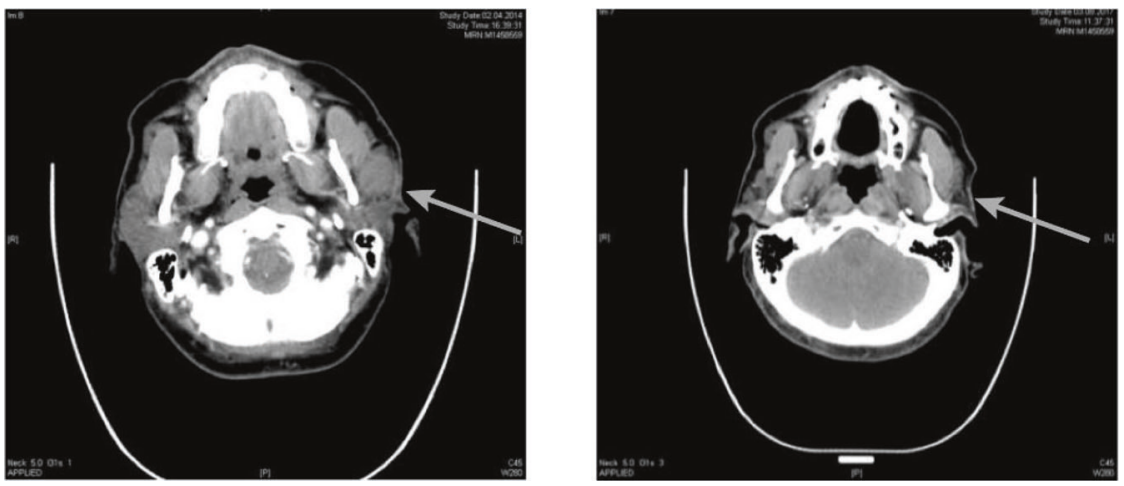

II
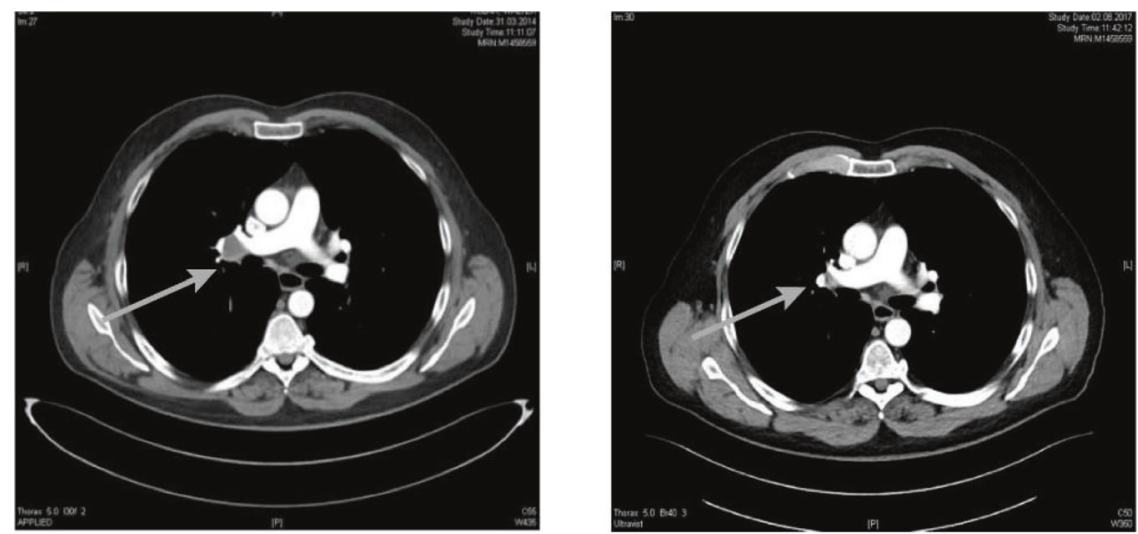

b

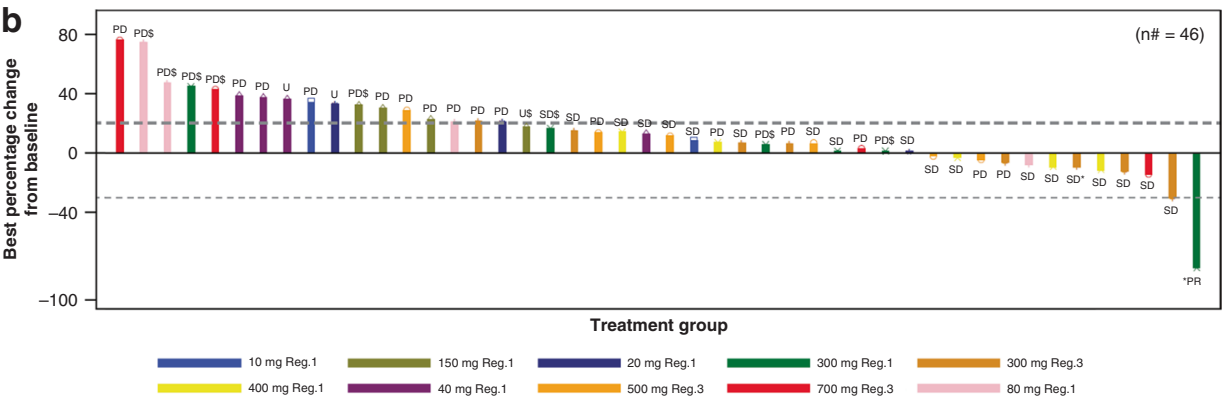

C

GDF-15 vs AUC $_{\text {last }}$

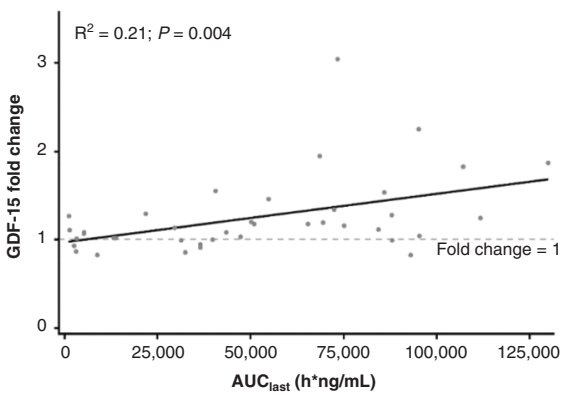

GDF-15 vs $C_{\max }$

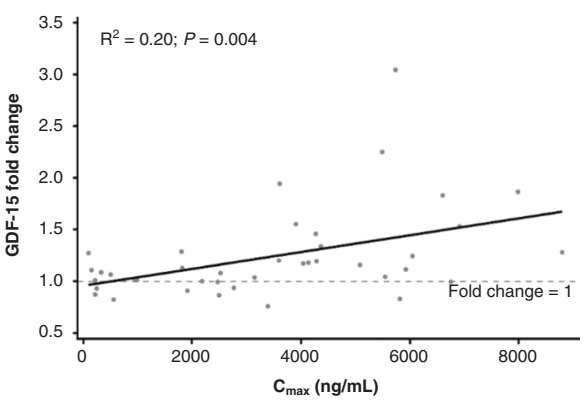

- Fold change from BL in GDF-15

ranging from 1.6 to $4.17 \mathrm{~h}$. Within the dose range of $10-700 \mathrm{mg}$, mean plasma exposures (area under the curve $\left[A \cup C_{\text {last }}\right.$ ] and maximum concentration $\left.\left[C_{\max }\right]\right)$ increased with increasing dose with no major deviation from dose proportionality, after single and repeated doses across all dose levels. Inter-patient variability expressed as a coefficient of variation was low to moderate for $A \cup C_{\text {last }}$ and $C_{\max }(12-63.3 \%$, refer to Supplementary Table S1).

p53 pathway activation by CGM097 was demonstrated in patients by induction of its downstream target GDF-15 in serum 
Fig. 2 Clinical efficacy and pharmacokinetic/pharmacodynamic data. a (I) Reduction of tumour size (brain CT) in a malignant skin melanoma patient treated with CGM097 (300 mg 3 qw) observing partial clinical response (left before, right after treatment). (II) Reduction of tumour size (thorax CT) in a malignant skin melanoma patient treated with CGM097 (300 mg 3 qw) observing partial clinical response (left before, right after treatment). b Best-percentage change from baseline in the sum of longest diameters with local mutational status and treatment group (full analysis set). $n=46$ is the number of patients for which the change in the total sum of the longest lesion diameters could be calculated, and best overall response as per investigators. Patients with baseline measurement only are not presented. Patients without any complete post baseline assessments are not included. Patients for which baseline and complete last assessments were available are included, even if these patients had missing assessments between baseline and last tumor assessments (U: unknown response, as per investigator assessments and in line with RECIST evaluation criteria). An asterisk symbol shows patients ongoing at the time of cut-off date. Symbol "\$" shows patients having P53 mutation. n\# number of subjects with baseline and post baseline CT scan results available. Reg. 1: 3 times weekly dosing, 4 weeks treatment cycle, continuous; Reg. 3: 3 times weekly dosing, 3 weeks treatment cycle; 2 weeks treatment, 1 week off treatment. c GDF-15-fold change from baseline versus $A U C_{\text {last }}$ and $C_{\max }$. Each point represents an individual patient's exposure and GDF-15 fold change. The continuous line represents linear regression with associated $\mathrm{R}^{2}$ and $P$ value. The dots are individual data, the full line displays a linear regression model through the data, with $R$-square indicating the proportion of the variability in the dependent variable (GDF-15) that is explained by the model, and $P$ value determining the statistical significance of the model. AUC last area under the curve from time $=0$ to last measurable concentration, BL baseline, $C_{\max }$ maximum concentration, GDF-15 growth differentiation factor 15 .

\begin{tabular}{|c|c|c|c|c|c|c|c|c|c|c|c|}
\hline & $\begin{array}{l}10 \mathrm{mg} \\
\text { Reg 1, } \\
N=3\end{array}$ & $\begin{array}{l}20 \mathrm{mg} \\
\text { Reg 1, } \\
N=4\end{array}$ & $\begin{array}{l}40 \mathrm{mg} \\
\text { Reg 1, } \\
N=4\end{array}$ & $\begin{array}{l}80 \mathrm{mg} \\
\operatorname{Reg} 1, \\
N=4\end{array}$ & $\begin{array}{l}150 \mathrm{mg} \\
\text { Reg 1, } \\
N=4\end{array}$ & $\begin{array}{l}300 \mathrm{mg} \\
\text { Reg 1, } \\
N=7\end{array}$ & $\begin{array}{l}400 \mathrm{mg} \\
\operatorname{Reg} 1, \\
N=5\end{array}$ & $\begin{array}{l}300 \mathrm{mg} \\
\text { Reg 3, } \\
N=8\end{array}$ & $\begin{array}{l}500 \mathrm{mg} \\
\text { Reg 3, } \\
N=6\end{array}$ & $\begin{array}{l}700 \mathrm{mg} \\
\text { Reg 3, } \\
N=6\end{array}$ & $\begin{array}{l}\text { All } \\
\text { patients, } \\
N=51\end{array}$ \\
\hline \multicolumn{12}{|l|}{ BOR } \\
\hline $\mathrm{CR}$ & 0 & 0 & 0 & 0 & 0 & 0 & 0 & 0 & 0 & 0 & 0 \\
\hline PR & 0 & 0 & 0 & 0 & 0 & $1(14.3)$ & 0 & 0 & 0 & 0 & $1(2.0)$ \\
\hline PD & $2(66.7)$ & $2(50.0)$ & $2(50.0)$ & $3(75.0)$ & $3(75.0)$ & $3(42.9)$ & $1(20.0)$ & $3(37.5)$ & $3(50.0)$ & $3(50.0)$ & $25(49.0)$ \\
\hline Unknown & 0 & $1(25.0)$ & $1(25.0)$ & 0 & $1(25.0)$ & $1(14.3)$ & 0 & 0 & 0 & $2(33.3)$ & $6(11.8)$ \\
\hline ORR & 0 & 0 & 0 & 0 & 0 & $1(14.3)$ & 0 & 0 & 0 & 0 & $1(2.0)$ \\
\hline $95 \% \mathrm{Cl}$ & {$[0.0,70.8]$} & {$[0.0,60.2]$} & {$[0.0,60.2]$} & {$[0.0,60.2]$} & {$[0.0,60.2]$} & {$[0.4,57.9]$} & {$[0.0,52.2]$} & {$[0.0,36.9]$} & {$[0.0,45.9]$} & {$[0.0,45.9]$} & {$[0.0,10.4]$} \\
\hline DCR & 1 (33.3) & $1(25.0)$ & $1(25.0)$ & $1(25.0)$ & 0 & $3(42.9)$ & $4(80.0)$ & $5(62.5)$ & $3(50.0)$ & 1 (16.7) & $2(39.2)$ \\
\hline
\end{tabular}

$B O R$ best overall response, $C l$ confidence interval, $D C R$ disease control rate, $O R R$ overall response rate, $P D$ progressive disease, $P R$ partial response, $R e g$ regimen. BOR was based on the investigator's assessment of disease status using RECIST 1.1.

$\mathrm{CR}$ and PR were confirmed by repeat assessments performed not less than 4 weeks after the criteria for the response was first met.

The $95 \% \mathrm{Cl}$ was calculated using the exact (Clopper-Pearson) interval.

Reg 1: 3qw dosing, 4-week treatment cycle, continuous; Reg 3: 3qw dosing, 3-week cycle; 2 weeks on treatment and 1 week off treatment.

(Fig. 2c). Increases in GDF-15 (fold change from baseline) were correlated with both CGM097 exposure and $C_{\max }(P$ value $=0.004$ for both).

\section{Pharmacokinetic and pharmacodynamic modelling}

The established clinical PK/PD models appropriately described the $\mathrm{PK}$, and the temporal relationship of drug action on platelets, and serum GDF-15. Matched individual observed vs. predicted time course for PK, platelets, and GDF-15, as well as goodness-of-fit plots, are shown in Fig. 3a and b, respectively. PK, platelet, and GDF-15 time course and variability parameters were estimated with good precision (Supplementary Table S2).

The PK of CGM097 was best described by a two-compartment model with a delayed first-order absorption (parameters Tlag and $\mathrm{ka}$ ) and a linear elimination. The model was parameterised by apparent oral clearance $(C L / F)$, apparent central volume of distribution (Vc/F), apparent inter-compartmental clearance $(Q / F)$, and apparent peripheral volume of distribution ( $V p / F)$. Population PK parameter estimates are reported in Supplementary Table S2. Population values were estimated to be $1.62 \mathrm{~L} / \mathrm{h}$ for $\mathrm{CL} / \mathrm{F}$ and $67 \mathrm{~L}$ for $\mathrm{Vc} / \mathrm{F}$ (inter-patient variability: $\mathrm{CL} / \mathrm{F}, 70 \%$; Vc/F, 35\%). The absorption rate constant $(k a)$ and absorption lag time (Tlag) were estimated to be $1.421 / \mathrm{h}$ and $0.39 \mathrm{~h}$, respectively.

To better understand CGM097-induced thrombocytopenia profiles, a semi-mechanistic myelosuppression model of platelet kinetics reproducing the hematopoietic maturation process was established by linking CGM097 plasma concentrations with observed blood platelet profiles. This model was modified from Friberg et al. ${ }^{14}$ Drug central compartment PK model concentrations $[C]$ were assumed to induce death of early proliferative hematopoietic cells via both a direct and an indirect drug effect through an effect compartment (Supplementary Fig. S6). The parameter estimates (PD platelet) are reported in Supplementary Table S2. Effect on early proliferative hematopoietic cells was associated with a mean maturation time (MMT) of $\sim 264 \mathrm{~h}$. Large IIV of 314 (CV\%) was estimated on the early cell killing parameter, defined by the term $s I P i$, reflecting the broad sensitivity across patients in response to bone marrow toxicity. All parameters were identifiable with relative standard errors below $30 \%$ and a low residual error (0.152), indicating that the model was able to adequately describe the population trends for long-term platelet profiles up to 20 months.

To characterise CGM097 exposure effect on GDF-15 kinetics and potential correlation with exposure effect on thrombocytopenia, a GDF-15 PK/PD model was developed. GDF-15 kinetics was best described by an indirect response model (Type III) with a drug effect on the zero-order input rate of GDF-15 production (kin). Baseline conditions were estimated independently of production and turnover (kout). The parameter estimates (PD GDF-15) are reported in Supplementary Table S2. All parameters were 

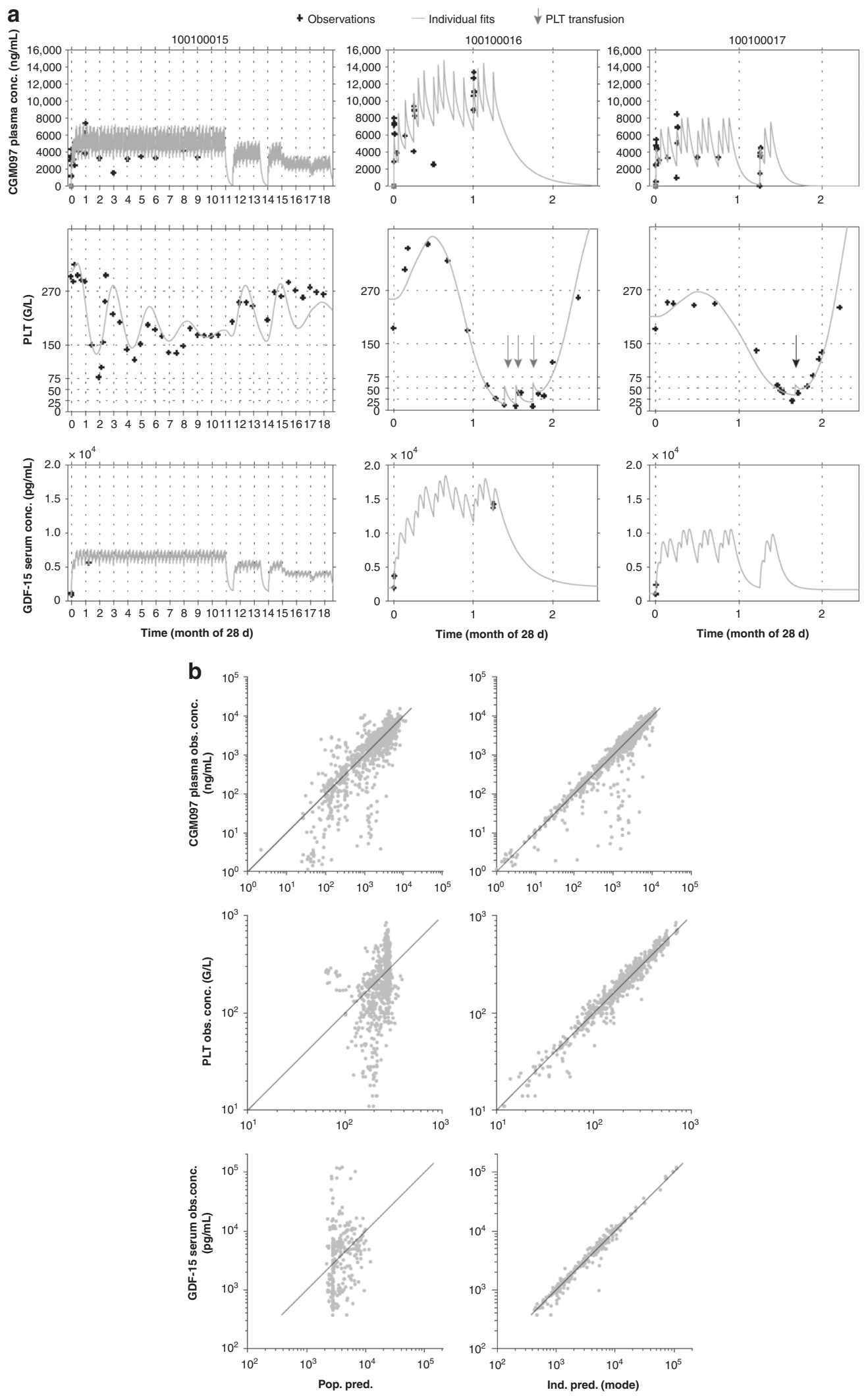

identifiable with relative standard errors below 20\% and a low residual error (0.191). The population value for GDF-15 at baseline (gdfZ) was estimated at $2780 \mathrm{pg} / \mathrm{mL}$ with a large IIV $(\mathrm{CV} \%=121)$. Intermittent 2 weeks on/1 week off dosing resulted in a return of GDF-15 level to baseline prior to the next treatment cycle. The correlation between model-derived individual drug effect on GDF15 production (denoted by $s / G i$ ) against the individual drug effect on immature hematopoietic cells (denoted by sIPi) is illustrated in Supplementary Fig. S8. These data, derived from 45 patients, with an $R^{2}$ of 0.51 , suggest that GDF-15 induction could be utilised as a 
Fig. 3 Pharmacokinetic and pharmacodynamic modelling of CGM097 concentrations, platelets count and GDF-15 kinetics. a Representative time course of the individual model fitted predictions versus observations for PK, PLT and GDF-15. Each vertical panel corresponds to a separate patient. Top, middle and bottom panels correspond, respectively, to individual CGM097 plasma concentrations (ng/ $\mathrm{mL})$, PLT counts $(\mathrm{G} / \mathrm{L})$ and GDF-15 serum concentrations $(\mathrm{pg} / \mathrm{mL}$ ) versus time profiles (day). Observed data are represented by black crosses and individual model fits by a continuous grey line. For the PLT time course, transfusion events are represented by vertical arrows. $\mathbf{b}$ Observed versus individual and population predicted for PK, PLT and GDF-15. The left and right panels represent observed versus predicted plots at the population level and individual level, respectively. The top, middle and bottom panels represent observed versus predicted plots for CGM097 plasma concentrations (ng/mL), PLT counts (G/L) and GDF-15 serum concentrations $(\mathrm{pg} / \mathrm{mL})$, respectively. conc concentration, d day, GDF-15 growth differentiation factor-15, Ind. pred. individual predicted, Obs. observed, PK phamacokinetic, PLT platelets, Pop. pred. population predicted. Dots represent the observed data.

prospective marker in the identification of patients at higher risk of developing delayed thrombocytopenia.

\section{DISCUSSION}

CGM097 administration in MDM2-amplified tumour models resulted in upregulation of p53 target genes, such as CDKN1A, BBC3, GDF-15 and HDM2 at the mRNA level, as previously published. In addition, preclinical efficacy experiments demonstrated that CGM097 could induce tumour regressions with daily dosing similar and consistent with other p53/MDM2 inhibitors ${ }^{5,20-22}$ However, here we report that similar preclinical regressions could be reached with an alternative dosing regimen, especially with the $3 q w$ regimen, which may have the best therapeutic index based on the low weekly dose and $C_{\text {ave-ss }}$ compared with the daily treatment regimen, and the low $C_{\text {max-ss }}$ compared with the 2qw and 3 days on/4 days off regimens.

These preclinical in vivo studies supported clinical exploration while providing preliminary evidence for dosing regimens. In the first-in-human phase I study of CGM097, adult patients with selected advanced solid tumours received different doses of CGM097 via two different dosing regimens: a 3qw continuous regimen and an alternative 3qw, 2 weeks on and 1 week off regimen, to allow bone marrow recovery. Clinical benefit was observed in 20 out of 51 patients. Of these, 19 patients had SD and a melanoma patient treated with CGM097 at $300 \mathrm{mg}$ (3qw dosing, continuous) achieved PR. No CR was reported. Of the patients with mutated p53 included in the trial (Fig. 2b), two-thirds progressed rapidly after the study start. In most of the enrolled patients, p53 sequencing was also reassessed using nextgeneration sequencing (NGS) with FoundationOne test, ${ }^{23}$ where the mutations in 9 patients were discovered. There are several potential reasons for these discrepancies in p53 sequencing. First, they could be due to the sensitivity and ability of different assays to detect mutations with lower allelic frequency. SNaPshot, ${ }^{24-26}$ Sanger ${ }^{27,28}$ and sequencing using AmpliChip Kit ${ }^{29}$ (with limit of detection ranging from $\sim 10-20 \%$ ) were utilised for sequencing prior to patient enrollment in the study, while NGS FoundationOne test (with a limit of detection of 5\%) was used retrospectively. The latter, in addition to higher sensitivity, offers improved range and depth of coverage. Second, a minimum sequencing of exons 5-8 in TP53 was required prior to enrollment, with the rationale that over $90 \%$ of mutations in p53 are found in the DNA-binding domain located between those exons and therefore mutations outside of these would be rare. $^{30}$ For FoundationOne, NGS sequencing reportable range encompasses all exons, and potentially provides another source for the discrepancy. Lastly, and perhaps the most likely reason for discrepancies in p53 mutation detection is the fact that sequencing was done on different biopsy samples. Tumour biopsies could be collected from different locations. In addition, archival tissue collected months before the study enrollment was allowed for screening. As the incidence of p53 mutations may increase with therapy-related genomic instability, above reasons may have contributed to the detection of $\mathrm{p} 53$ mutations after the patient has been enrolled in the study. ${ }^{31}$
A major concern with p53-reactivating therapies is its effect on normal cells, which includes the stabilisation of p53 resulting in increased apoptosis in these cells. In a clinical trial of RG7112 in liposarcoma, the most common toxicity was haematological, with $30 \%$ of patients experiencing grade 4 neutropenia, and $15 \%$ experiencing prolonged grade 4 thrombocytopenia. ${ }^{32-34}$ However, whether the haematologic toxicity correlated with prior exposure to genotoxic therapies is unknown. There were also reports of an increased incidence of p53 mutations following prolonged Nutlin$3 a$ exposure ${ }^{35}$ and concerns about this effect on the development of new cancers. ${ }^{36}$ In this study, the most frequently reported treatment-related AEs included thrombocytopenia, nausea, leukopenia, vomiting, and fatigue, and the most common treatmentrelated grade 3 or 4 AEs were thrombocytopenia, lymphopenia and neutropenia. No DLTs were observed in the range of tested doses within the DLT period of 4 weeks, as the haematologicalrelated events were generally observed after day 28 (cycle 1) due to the delayed onset of myelosuppression relative to the start of drug treatment. The most frequently occurring treatment-related grade $\geq 3 \mathrm{AE}$ was thrombocytopenia. Since HDM2 plays a role in normal hematopoiesis, this effect was considered as on-target and consistent with other HDM2-antagonist agents. ${ }^{32-34}$

MTD was not reached. The study was halted prematurely due to a strategic decision by Novartis to develop HDM201, a secondgeneration HDM2 inhibitor. In comparison to CGM097, HDM201 demonstrates improved preclinical properties. Even though both HDM201 and CGM097 are highly potent and selective inhibitors of HDM2-p53 interaction, with IC50 values of $0.2 \mathrm{nM}$ versus $1.5 \mathrm{nM}$, respectively, HDM201 showed improved physicochemical properties $^{2}$ and an improved PK profile allowing continuous low-dose and pulse high-dose dosing regimen. ${ }^{18}$ Therefore, after a thorough comparison of the preclinical and clinical data from both HDM2 inhibitor programmes, Novartis decided to prioritise the clinical development of HDM201.

The ability to quantitatively assess drug-induced haematological toxicity is of great value for dose optimisation, administration schedules, and in predicting complications for patients who undergo prolonged periods of myelosuppression. Here, we developed a semi-mechanistic thrombocytopenia model for CGM097, and showed that the model can adequately describe the relationship between drug concentrations and the long-term time course of platelets following the administration of CGM097. Appropriate description of platelet profiles required the model to have both a direct and an indirect drug-induced depletion of immature cells, and a drug effect on systemic regulation. The model was able to describe the longitudinal time course of platelet changes associated with up to 20 months of treatment duration. Clinically, one of the major toxicities associated with CGM097 administration was found to be the delayed onset of thrombocytopenia. The delayed onset of CGM097-induced thrombocytopenia and the long platelet recovery time were addressed through an adapted dosing strategy and guided by the thrombocytopenia model, including higher dose treatment followed by a drug holiday period to maintain the total dose per cycle. Switching to an intermittent dosing schedule resulted in a manageable platelet 
profile while still achieving a similar average dose per cycle compared to a continuous schedule. This is reflected by the relatively long model-derived estimate for MMT on circulating platelets of $\sim 264 \mathrm{~h}$. Unlike previously reported shorter MMT values for classic cytotoxic chemotherapy agents, ${ }^{14}$ the estimated MMT value in this study was consistent with that of other HDM2 inhibitors and therefore likely to be a common characteristic of HDM2 inhibitors. ${ }^{37}$ In addition, large variability in developing CGM097-induced thrombocytopenia was observed among patients. These clinical effects were further reflected by the very large model-derived inter-individual variability of the drug thrombocytopenia potency parameter at $\sim 314 \%$. Indeed, the ability to predict the likelihood that a patient will develop delayed, drug-induced thrombocytopenia early enough would allow proactive toxicity management of the patient. The PK/PD modelderived individual drug potency on GDF-15 production on day 1 was associated with the drug thrombocytopenia potency, raising the possibility to use GDF-15 induction as a prospective marker in the identification of patients at a higher risk of developing delayed thrombocytopenia (Supplementary Fig. S1). The link of circulating GDF-15 as an early predictive marker of delayed thrombocytopenia related to CGM097 or other HDM2 inhibitors would require further investigations beyond the exploratory analysis of this study. Overall, this work provides an integrated quantitative understanding of thrombocytopenia and the GDF-15 biomarker changes in response to HDM2 inhibitors, with potential use in dosing regimen optimisation and patient benefit.

While CGM097 did not show a clinically significant number of tumour regressions, more patients rather experienced disease stabilisations, we were however able to reactivate p53 at therapeutically relevant doses. This study provides a relevant reference for potential drug combination studies that may hold greater promise for MDM2 inhibitors than monotherapies. This study further confirms that for p53-inducing drugs, the therapeutic window, especially in the context of delayed haematological toxicity, may require highly specific scheduling as well as predictive PD markers to improve patient benefit while mitigating the safety risk of severe thrombocytopenia. In conclusion, although this study was prematurely discontinued, we have developed the methods to improve the dosing of MDM2 inhibitor using extensive PK/PD modelling. The important learnings derived from the CGM097X2101 first-in-human study are supporting the new generation MDM2 inhibitors in development which are currently tested in clinics.

\section{ACKNOWLEDGEMENTS}

The authors thank the patients participating in this clinical trial, and their families. The authors thank the staff at each participating Institution that assisted with the study. We thank Jutta Beyer, Senior Global Program Manager and Giorgia Clementi, Global Program Manager for providing the technical inputs and driving the manuscript. The authors would also like to thank Siyan $\mathrm{Xu}$, Senior Principal Pharmacometrician, Novartis Institutes for BioMedical Research for providing additional statistical support for the study. We also thank Dr. Anamika Gulati, PhD and Haritha Nekkanti PharmD of Novartis Healthcare Pvt Ltd, Hyderabad, India for medical editorial assistance with the manuscript. Presented in part as Poster presentations (2734 and 1273) at European Society for Medical Oncology (ESMO) 2016; Copenhagen, Denmark, October 7 to 11, 2016.

\section{AUTHOR CONTRIBUTIONS}

Conception and design-J.W. and L.V.B. Development of methodology-J.W., L.V.B., N.G., C.M., S.F., E.H., A.J., S.B., S.J., C.F. and F.H.P. Acquisition of the data-S.B., G.D., R.D., D.T. and P.C. Analysis and interpretation of the data-all authors. Writing, review and/or revision of the manuscript—all authors. Study supervision-Novartis.

\section{ADDITIONAL INFORMATION}

Ethics approval and consent to participate The study protocol and all amendments were reviewed by the Independent Ethics Committee or Institutional
Review Board for each centre. The study was conducted according to the ethical principles of the Declaration of Helsinki. All animal experiments were performed according to procedures covered by permit number BS-1975 issued by the Cantonal Veterinary Office, Basel, Switzerland, and in strict adherence to the Federal Animal Protection Act and the Federal Animal Protection Code.

\section{Consent to publish Not applicable.}

Data availability Novartis will not provide access to patient-level data if there is a reasonable likelihood that individual patients could be re-identified. Phase 1 studies, by their nature, present a high risk of patient re-identification; therefore, patient individual results for phase 1 studies cannot be shared. In addition, clinical data, in some cases, have been collected subject to contractual or consent provisions that prohibit transfer to third parties. Such restrictions may preclude granting access under these provisions. Where co-development agreements or other legal restrictions prevent companies from sharing particular data, companies will work with qualified requestors to provide summary information where possible.

Competing interests S.B. has received honoraria from Novartis, Pfizer, Bayer, PharmaMar and GlaxoSmithKline; served in advisory or consultancy roles for Blueprint Medicines, Bayer, Lilly, Deciphera, Exelixis, Janssen-Cilag, Plexxikon and Nanobiotix; received institutional research funding from Blueprint Medicines, Novartis, and Incyte; and received travel and accommodation funding from PharmaMar. G.D. reports grants, non-financial support and personal fees from Novartis during the conduct of the study. Outside of this work, the following disclosures are reported: grants and personal fees from Bayer, personal fees from Pfizer, personal fees and non-financial support from Roche/Genentech, grants and personal fees from Epizyme, grants and personal fees from LOXO Oncology, grants, personal fees, and non-financial support from AbbVie, grants, personal fees, and nonfinancial support from GlaxoSmithKline, grants and personal fees from Janssen, personal fees and non-financial support from PharmaMar, personal fees from ZioPharm, grants, personal fees and non-financial support from Daiichi-Sankyo, grants and personal fees from Adaptimmune, personal fees from Ignyta, personal fees from Mirati, personal fees from EMD Serono, personal fees from Sanofi, personal fees and non-financial support from ICON PLC, personal fees and non-financial support from WCG/ARSENAL, personal fees from Polaris Pharmaceuticals, personal fees from MJ Hennessey/OncLive, personal fees from Medscape, grants and nonfinancial support from the Dr. Miriam and Sheldon G. Adelson Medical Research Foundation, personal fees and "minor equity" from G1 Therapeutics, minor equity, personal fees, non-financial support, and other from CARIS Life Sciences, minor equity from Bessor Pharmaceuticals, minor equity from ERASCA Pharmaceuticals, minor equity and personal fees from RELAY Therapeutics, minor equity and personal fees from Caprion/HistoGeneX, minor equity, personal fees, non-financial support, and other from Blueprint Medicines (where he serves as a member of the Board of Directors), personal fees and member of the Board of Directors (until Oct 2019) of Merrimack Pharmaceuticals, and personal fees, minor equity as a member of the Board of Directors of Translate BIO, all outside the submitted work. S.J. was an employee of Novartis Oncology at the time the study was performed. S.J. is now an employee of Idorsia Pharmaceuticals Ltd. In addition, S.J. has patents WO/2015/ 097622 and WO/2014/020502 issued. R.D. has intermittent, project focused consulting and/or advisory relationships with Novartis, Merck Sharp \& Dhome (MSD), Bristol-Myers Squibb (BMS), Roche, Amgen, Takeda, Pierre Fabre, Sun Pharma, Sanofi, Catalym, Second Genome outside the submitted work. N.G. was an employee of Novartis Pharma AG at the time the study was performed, and owning shares of Novartis Pharma AG. N.G. is now an employee of F. Hoffmann-La Roche AG. D.T. is a senior consultant at the National Cancer Centre Singapore. D.T. has consulting and/or advisory relationships with Novartis, Bayer, Boehringer Ingelheim, Celgene, AstraZeneca, Eli-lilly, LOXO, Pfizer, Takeda and Merrimack; Honoraria from Boehringer Ingelheim, Merck, Roche and Pfizer; Research Grants from Novartis, Bayer, AstraZeneca, GlaxoSmithKline, outside the submitted work. A.J. is an employee of Novartis Pharma AG owning shares of Novartis Pharma AG. C.M. is an employee of Novartis Pharma AG owning shares of Novartis Pharma AG. S.F. is an employee of Novartis Pharma AG owning shares of Novartis Pharma AG. L.V.B. is an employee of Novartis Pharma AG owning shares of Novartis Pharma AG. E.H. is an employee of Novartis Pharma AG owning shares of Novartis AG. F.H.P. is an employee of Novartis Pharma AG owning shares of Novartis AG. C.F. is an employee of Novartis Pharma AG owning shares of Novartis Pharma AG. J.W. was an employee of Novartis Oncology at the time the study was performed and is holding shares of Novartis Pharma AG. J.W. is now an employee of ADCT Therapeutics SA. P.C.: Honoraria: Amgen, Astra-Zeneca, Blueprint, EMD Serono, Roche/Genentech, Novartis; Travel accommodation: AstraZeneca, BMS, EMD Serono, MSD, Novartis; Research support to institution: Abbvie, Bayer, Blueprint, BMS, GSK, Janssen, Lilly, Loxo, Novartis, Roche/Genentech, Taiho. P.C. is a member of the Editorial Board of the British Journal of Cancer. 
Funding information The study was sponsored by Novartis Pharma AG. Open Access funding enabled and organized by Projekt DEAL.

Supplementary information The online version contains supplementary material available at https://doi.org/10.1038/s41416-021-01444-4.

Publisher's note Springer Nature remains neutral with regard to jurisdictional claims in published maps and institutional affiliations.

\section{REFERENCES}

1. Khoo, K. H., Verma, C. S. \& Lane, D. P. Drugging the p53 pathway: understanding the route to clinical efficacy. Nat. Rev. Drug Discov. 13, 217-236 (2014).

2. Holzer, P., Masuya, K., Furet, P., Kallen, J., Valat-Stachyra, T., Ferretti, S. et al. Discovery of a dihydroisoquinolinone derivative (NVP-CGM097): a highly potent and selective MDM2 inhibitor undergoing phase 1 clinical trials in p53wt tumors. J. Med. Chem. 58, 6348-6358 (2015).

3. Vaupel, A., Holzer, P., Ferretti, S., Guagnano, V., Kallen, J., Mah, R. et al. In vitro and in vivo characterization of a novel, highly potent p53-MDM2 inhibitor. Bioorg. Med. Chem. Lett. 28, 3404-3408 (2018).

4. Weisberg, E., Halilovic, E., Cookke, V. G., Nonami, A., Ren, T., Sanda, T. et al. Inhibition of wild-type p53-expressing AML by the novel small molecule HDM2 inhibitor CGM097. Mol. Cancer Ther. 14, 2249-2259 (2015).

5. Jeay, S., Gaulis, S., Ferretti, S., Bitter, H., Ito, M., Valat, T. et al. A distinct p53 target gene set predicts for response to the selective p53-HDM2 inhibitor NVP-CGM097. elife 4, e06498 (2015).

6. Barretina, J., Caponigro, G., Stransky, N., Venkatesan, K., Margolin, A. A., Kim, S. et al. The Cancer Cell Line Encyclopedia enables predictive modelling of anticancer drug sensitivity. Nature 483, 603-607 (2012).

7. Ladanyi, M., Cha, C., Lewis, R., Jhanwar, S. C., Huvos, A. G. \& Healey, J. H. MDM2 gene amplification in metastatic osteosarcoma. Cancer Res. 53, 16-18 (1993).

8. Olivier, M., Hollstein, M. \& Hainaut, P. TP53 mutatioins in human cancers: origins, consequences, and clinical use. Cold Spring Harb. Perspect. Biol. 2, a001008 (2010).

9. Yu, Z., Zhang, B., Cui, B., Wang, Y., Han, P. \& Wang, X. Identification of spliced variants of the proto-oncogene HDM2 in colorectal cancer. Cancer 118, 1110-1118 (2012).

10. Singer, E. A., Bratslavsky, G., Linehan, W. M. \& Srinivasan, R. Targeted therapies for non-clear renal carcinomas. Target Oncol. 5, 119-129 (2010).

11. Vetter, T. R. Descriptive statistics: Reporting the answers to the 5 basic questions of who, what, why, when, where, and a sixth, so what? Anesth. Analg. 125, 1797-1802 (2017).

12. Sheiner, L. B. \& Ludden, T. M. Population pharmacokinetics/dynamics. Annu. Rev. Pharm. Toxicol. 32, 185-209 (1992)

13. Deylon, B., L. M. \& Moulines, E. Convergence of a stochastic approximation version of the EM algorithm. Ann. Stat. 27, 34 (1999).

14. Friberg, L. E., Henningsson, A., Maas, H., Nguyen, L. \& Karlsson, M. O. Model of chemotherapy-induced myelosuppression with parameter consistency across drugs. J. Clin. Oncol. 20, 4713-4721 (2002).

15. Sharma, A. \& Jusko, W. J. Characteristics of indirect pharmacodynamic models and applications to clinical drug responses. Br. J. Clin. Pharm. 45, 229-239 (1998).

16. Guerreiro, N., Jullion, A., Ferretti, S., Fabre, C. \& Meille, C. Translational modeling of anticancer efficacy to predict clinical outcomes in a first-in-human phase 1 study of MDM2 inhibitor HDM201. AAPS J. 23, 28 (2021).

17. Verhaegen, M., Checinska, A., Riblett, M. B., Wang, S. \& Soengas, M. S. E2F1dependent oncogenic addiction of melanoma cells to MDM2. Oncogene 31, 828-841 (2012).

18. Jeay, S., Feretti, S., Holzer, P., Fuchs, J., Chapeau, E. A., Wartmann, M. et al. Dose and schedule determine distinct molecular mechanisms underlying the efficacy of the p53-MDM2 inhibitor HDM201. Cancer Res. 78, 6257-6267 (2018).

19. Zhao, Y., Aguilar, A., Bernard, D. \& Wang, S. Small-molecule inhibitors of the MDM2-p53 protein-protein interaction (MDM2 Inhibitors) in clinical trials for cancer treatment. J. Med. Chem. 58, 1038-1052 (2015).

20. Tovar, C., Graves, B., Packman, K., Filipovic, Z., Higgins, B., Xia, M. et al. MDM2 small-molecule antagonist RG7112 activates p53 signaling and regresses human tumors in preclinical cancer models. Cancer Res. 73, 2587-2597 (2013).
21. Canon, J., Osgood, T., Olson, S. H., Saiki, A. Y., Robertson, R., Yu, D. et al. The MDM2 inhibitor AMG 232 demonstrates robust antitumor efficacy and potentiates the activity of p53-inducing cytotoxic agents. Mol. Cancer Ther. 14, 649-658 (2015).

22. Wang, S., Sun, W., Zhao, Y., McEachern, D., Meaux, I., Barriere, C. et al. Sar405838: an optimized inhibitor of MDM2-p53 interaction that induces complete and durable tumor regression. Cancer Res. 74, 5855-5865 (2014).

23. Inc, F. M. Foundationone $\mathrm{CDx}^{\mathrm{TM}}$ technical information. https://www.accessdata. fda.gov/cdrh_docs/pdf17/P170019C.pdf (2017).

24. O'Donnell, E., Mahindra, A., Yee, A., Nardi, V., Birrer, N., Horick, N. et al. Clinical grade "SNaPshot" genetic mutation profiling in multiple myeloma. EBioMedicine 2, 71-73 (2015).

25. Penson, R. T., Sales, E., Sullivan, L., Borger, D. R., Krasner, C. N., Goodman, A. K. et al. A SnaPshot of potentially personalized care: Molecular diagnostics in gynecologic cancer. Gynecol. Oncol. 141, 108-112 (2016).

26. Sarasqueta, A. F., Moerland, E., de Bruyne, H., de Graaf, H., Vrancken, T., van Lijnschoten, G. et al. SNaPshot and StripAssay as valuable alternatives to direct sequencing for KRAS mutation detection in colon cancer routine diagnostics. J. Mol. Diagn. 13, 199-205 (2011).

27. Sanger, F. \& Coulson, A. R. A rapid method for determining sequences in DNA by primed sunthesis with DNA polymerase. J. Mol. Biol. 94, 441-448 (1975).

28. Rohlin, A., Wernersson, J., Engwall, Y., Wiklund, L., Björk, J. \& Margareta, N. Parallel sequencing used in detection of mosaic mutations: comparison with four diagnostic DNA screening techniques. Hum. Mutat. 30, 1012-1020 (2009).

29. Chiaretti, S., Tavolaro, S., Marinelli, M., Messina, M., Giudice, I. D., Mauro, F. R. et al. Evaluation of TP53 mutations with the AmpliChip p53 research test in chronic lymphocytic leuukemia: correlation with clinical outcome and gene expression profiling. Genes Chromosomes Cancer 50, 263-274 (2011).

30. Greenblatt, M. S., Bennett, W. P., Hollstein, M. \& Harris, C. C. Mutations in the p53 tumor suppressor gene: clues to cancer etiology and molecular pathogenesis. Cancer Res 54, 4855-4878 (1994).

31. Barbosa, K., Li, S., Adams, P. D. \& Deshpande, A. J. The role of TP53 in acute myeloid leukemia: challenges and opportunities. Genes Chromosomes Cancer 58, 875-888 (2019).

32. Iancu-Rubin, C., Mosoyan, G., Glenn, K., Gordon, R. E., Nichols, G. L. \& Hoffman, R. Activation of $\mathrm{p} 53$ by the MDM2 inhibitor RG7112 impairs thrombopoiesis. Exp. Hematol. 42, 137-145 e135 (2014).

33. Ray-Coquard, I., Blay, J. Y., Italiano, A., Le Cesne, A., Penel, N., Zhi, J. et al. Effect of the MDM2 antagonist RG7112 on the p53 pathway in patients with MDM2amplified, well-differentiated or dedifferentiated liposarcoma: an exploratory proof-of-mechanism study. Lancet Oncol. 13, 1133-1140 (2012).

34. Wagner, A. J., Banerji, U., Mahipal, A., Somaiah, N., Hirsch, H., Fancourt, C. et al. Phase I trial of the human double minute 2 inhibitor MK-8242 in patients with advanced solid tumors. J. Clin. Oncol. 35, 1304-1311 (2017).

35. Aziz, M. H., Shen, H. \& Maki, C. G. Acquisition of $p 53$ mutations in response to the non-genotoxic p53 activator nutlin-3. Oncogene 30, 4678-4686 (2011).

36. Francoz, S., Froment, P., Bogaerts, S., De Clercq, S., Maetens, M., Doumont, G. et al. MDM4 and MDM2 cooperate to inhibit p53 activity in proliferating and quiescent cells in vivo. Proc. Natl Acad. Sci. USA 103, 3232-3237 (2006).

37. Tisato, V., Voltan, R., Gonelli, A., Secchiero, P. \& Zauli, G. MDM2/X inhibitors under clinical evaluation: perspectives for the management of hematological malignancies and pediatric cancer. J. Hematol. Oncol. 10, 133 (2017).

Open Access This article is licensed under a Creative Commons Attribution 4.0 International License, which permits use, sharing, adaptation, distribution and reproduction in any medium or format, as long as you give appropriate credit to the original author(s) and the source, provide a link to the Creative Commons license, and indicate if changes were made. The images or other third party material in this article are included in the article's Creative Commons license, unless indicated otherwise in a credit line to the material. If material is not included in the article's Creative Commons license and your intended use is not permitted by statutory regulation or exceeds the permitted use, you will need to obtain permission directly from the copyright holder. To view a copy of this license, visit http://creativecommons. org/licenses/by/4.0/.

(c) The Author(s) 2021 